\title{
Norois
}

Environnement, aménagement, société

$243 \mid 2017$

Capital environnemental et espaces emblématiques

\section{Environnement, conflits et tractations entre divers acteurs ruraux au Québec : une mobilisation inégale du capital environnemental?}

Environment, conflicts and negotiations between various rural actors in Quebec: An unequal mobilization of environmental capital ?

\section{Myriam Simard}

\section{OpenEdition \\ Journals}

Édition électronique

URL : https://journals.openedition.org/norois/6111

DOI : $10.4000 /$ norois.6111

ISBN : 978-2-7535-6537-7

ISSN : 1760-8546

Éditeur

Presses universitaires de Rennes

\section{Édition imprimée}

Date de publication : 2 novembre 2017

Pagination : 111-126

ISBN : 978-2-7535-6465-7

ISSN : 0029-182X

Référence électronique

Myriam Simard, «Environnement, conflits et tractations entre divers acteurs ruraux au Québec : une mobilisation inégale du capital environnemental ? », Norois [En ligne], 243 | 2017, mis en ligne le 20 septembre 2019, consulté le 13 janvier 2022. URL : http://journals.openedition.org/norois/6111 ; DOI : https://doi.org/10.4000/norois.6111 


Nö́ris a

\title{
Environnement, conflits et tractations entre divers acteurs ruraux au Québec : une mobilisation inégale du capital environnemental?
}

\author{
Environment, Conflicts and Negotiations between Various Rural Actors in Quebec: \\ An Unequal Mobilization of Environmental Capital?
}

\begin{abstract}
Myriam Simard
Professeure et Directrice du Groupe de recherche sur la migration ville/campagne et les néoruraux (http://uwww.neoruraux. ucs.inrs.ca/), Institut National de la Recherche Scientifique (INRS), Université du Québec, Centre Urbanisation, Culture et Société - 385 Sherbrooke Est, MontréAL, Québec, Canada, H2X 1E3. (myriam.simard@ucs.inrs.ca)
\end{abstract}

En hommage à mon conjoint et citoyen engagé, Raymond Asselin, décédé le 5 mars 2016

Résumé : Les enjeux environnementaux étant les plus conflictuels dans le Québec rural, l'objectif de l'article est d'en examiner les rapports de pouvoir entre divers groupes rivaux, à la lumière du capital environnemental. Ce concept, inspiré de la théorie de Bourdieu, apparait comme une clef explicative de la complexité de ces rapports, surtout lorsqu'il est associé aux autres ressources et capitaux des acteurs en présence. Pour repérer la dynamique globale de ces luttes de pouvoir, marquées par des fractures, des tractations et des compromis fragiles, les enjeux environnementaux ont été couplés à ceux politiques. Car, derrière les conflits d'usage des espaces naturels transparaissent des enjeux politiques de gestion municipale et d'aménagement territorial. Cette juxtaposition d'enjeux oblige les acteurs, lors de leur mobilisation du capital environnemental, à s'investir aussi dans le processus décisionnel local et à déployer leurs autres atouts pour s'imposer. S'affrontent alors des protagonistes aux ressources inégales qui préconisent deux conceptions contradictoires du développement rural, avec des valeurs, représentations, pratiques et intérêts opposés. Les données proviennent d'une analyse comparative sur les interactions à l'environnement de quatre groupes d'acteurs dans deux territoires contrastées au Québec : les néoruraux, ruraux de longue date, dirigeants d'organismes et élus municipaux. Ce sont davantage les néoruraux et les élus municipaux qui s'affrontent, chacun utilisant leur capital environnemental respectif pour gagner. Selon les circonstances, ils auront comme alliés soit les ruraux de longue date, soit les dirigeants d'organismes locaux.

Abstract: The preservation of the environment represents one of the most challenging topics in rural Quebec. The aim of this paper is to explore the power relations between various rival groups, in light of their environmental capital. This concept, based on Bourdieu's theory, seems to be a key explaining the complexity of these relations, especially when associated with other resources and capitals. To identify the overall dynamics of these relations, characterized by rifts and fragile compromises, links were established between environmental issues and political issues - for behind conflicts over the use of natural spaces lie political issues connected with municipal management and planning. This juxtaposition of issues requires actors, when they mobilize their environmental capital, to also invest in local decisionmaking processes and deploy their other assets in order to impose their values. A confrontation emerges between protagonists with unequal resources, advocating two contradictory concepts of rural development, with opposing values, representations, practices and interests. The data come from a comparative analysis of four groups of actors' interactions with the environment in two contrasting Quebec territories: the rural newcomer, the long-time rural inhabitant, the leaders of local organizations, and elected municipal officials. The confrontation occurs primarily between the rural newcomers and the elected officials, each using their respective environmental capital to triumph over the other. Depending on the circumstances, their allies may be either the long-time rural inhabitants or the leaders of local organizations. 
Mots clés : campagne - conflits d'usage - néoruraux - capital environnemental - enjeux politiques et environnementaux - comparaison entre acteurs ruraux - Québec (Canada)

Keywords: countryside - conflicts of rural uses - neorural populations (rural newcomers) - environmental capital - political and environmental issues - comparison between rural actors - Quebec (Canada)

\section{INTRODUCTION}

Les campagnes québécoises connaissent une recomposition sociodémographique avec l'arrivée de nouvelles populations rurales depuis la décennie $1990^{1}$. Se pose la question de leur cohabitation avec les autres ruraux d'installation plus ancienne lorsque des enjeux cruciaux apparaissent. Ontils des relations solidaires ou plutôt d'opposition? Interagissent-ils avec les décideurs locaux avec confiance ou méfiance? Quelles sont leurs stratégies respectives pour faire valoir leurs points de vue dans les débats sur ces enjeux? Ceci soulève le sujet des rapports de pouvoir entre les divers acteurs ruraux. Sont-ils tous égaux lors des négociations sur ces enjeux?

Appréhender ces nouvelles relations pose un défi car elles demeurent peu documentées dans les sciences sociales en raison d'un désintérêt pour l'analyse des complexités du pouvoir dans les sociétés rurales (Cloke et Goodwin, 1993; Gosnell et Abrams, 2011). En dépit d'une littérature internationale substantielle sur la néoruralité, les interactions sociales ne sont que fragmentaires, étant souvent réduites aux seuls conflits entre néoruraux ${ }^{2}$ et ruraux de longue date de classes sociales distinctes (Cloke et Thrift, 1987; Boyle et Halfacree, 1998). Les acteurs décisifs tels les décideurs sont habituellement ignorés (Simard, 2011).

L'objectif de cet article est d'offrir une vue globale des interactions et des tractations concernant l'enjeu le plus conflictuel dans le Québec rural, à savoir environnemental. Il s'agit d'approfondir ces rapports en comparant quatre catégories d'acteurs,

1. La migration de la ville vers la campagne s'amorce plus lentement au Québec qu'en Europe, de sorte que les études québécoises sur cet aspect sont plus récentes (Simard, 2007). Voir un no spécial consacré à ce sujet où une comparaison entre la France et le Québec est présentée en introduction (Simard, 2011).

2. Le terme «néorural » est davantage utilisé au Québec qu’en France pour nommer les nouveaux résidents. Il n'a pas la même connotation suite aux contestations françaises de mai 1968, car il n’implique pas une rupture avec la société de consommation et la vie urbaine. Voir le tableau 1 pour les définitions des quatre catégories d'acteurs. soit les néoruraux, les ruraux de longue date et les décideurs formés des dirigeants d'organismes et des élus municipaux. Leurs jeux de pouvoir et les principales stratégies utilisées, dont la réquisition de réseaux et atouts variés, sont scrutés. Or, à l'examen des enjeux environnementaux, une autre facette complémentaire ressort, à savoir les enjeux politiques. Car, pour dégager une vue holistique des dynamiques de pouvoir/contre-pouvoir, il faut explorer tant les terrains d'ententes et/ou de disputes, que les valeurs, intérêts et représentations associés aux deux enjeux, ainsi que les pratiques et pourparlers de chacun des acteurs pour parvenir à leurs fins.

Dès lors, on constate les rapports inégaux des acteurs lors des négociations sur ces enjeux. Comment les interpréter? Avec quel outil d'analyse? Inspiré de Bourdieu, le concept de capital environnemental peut s'avérer utile pour clarifier ces rapports contrastés à l'environnement, dans le cadre de la multifonctionnalité des campagnes. Révélateur de tensions inconciliables, il semble pertinent pour offrir d'éventuelles pistes d'interprétation valables de ces interactions et jeux de pouvoir.

L'article comporte trois parties. La première présente les deux repères théoriques de l'étude, les conflits d'usage de la campagne et le concept de capital environnemental. La deuxième expose la méthodologie. La troisième présente les résultats sous l'angle du capital environnemental pour interpréter les conflits touchant les enjeux environnementaux et politiques dans le Québec rural. L'exemple d'un projet de carrière dans un des territoires étudiés vient clore cette partie par une illustration récente de la mobilisation du capital environnemental. Somme toute, ce sont deux principales conceptions contradictoires du développement et de la protection de l'environnement qui ressortent de notre analyse. En conclusion, la nécessité de poursuivre les réflexions sur le concept de capital environnemental est affirmée dans le contexte d'in- 
tensification des préoccupations écologiques et des luttes environnementales du XIX $x^{\mathrm{e}}$ siècle.

\section{REPÈRES THÉORIQUES : LES USAGES CONTRADICTOIRES DE LA CAMPAGNE SOUS L'ANGLE DU CAPITAL ENVIRON- NEMENTAL}

\section{Conflits d'usage de la campagne et intérêts différenciés}

Afin de comprendre les interactions et les tractations entre les quatre groupes de ruraux sur les enjeux environnementaux, deux corpus théoriques de tradition distincte, l'un français et l'autre anglosaxon, furent utilisés pour leurs multidisciplinarités et complémentarités au sujet des divers usages de l'espace rural. Ils approfondissent tant les causes des conflits et des alliances que leurs significations dans la répartition du pouvoir concernant les enjeux environnementaux, ceux-ci étant prépondérants dans la littérature comparativement aux autres enjeux (Abram et al., 2012).

Retenons que les usages de l'espace rural se déclinent en quatre fonctions souvent concurrentes : productive, résidentielle, récréative et de préservation (Perrier-Cornet, 2002 ; Slee, 2005). Cette multifonctionnalité est associée à ce que Perrier-Cornet nomme la «publicisation des campagnes » due à la popularité du « cadre de vie rural » et de la «nature » et bien illustrée par la migration d'agrément ou de style de vie (Cadieux et Hurley, 2011; Martin et al., 2012). Cette multifonctionnalité génère des conflits pour l'utilisation et le contrôle de l'espace rural par des usagers rivaux tels les agriculteurs, les néoruraux ou les touristes. D’où la notion de conflits d'usage et de voisinage qui a catalysé une abondante littérature (Kirat et Torre, 2006).

S'arrimant aux diverses fonctions, ces conflits sont liés soit à la production d'activités, soit à la consommation de l'espace rural à des fins résidentielles ou récréatives, soit à la protection de son écosystème. Ils renvoient à des valeurs, opinions, intérêts et représentations hétéroclites sur le milieu rural et l'environnement. Paradoxalement, ils assurent une certaine cohésion sociale par la recherche de compromis lors des discussions sur les orientations du développement rural.
Ces conflits interpellent le pouvoir public local, dans son rôle de concertation et d'aménagement territorial, afin de normaliser la coexistence des usages (Brunori et Rossi, 2007 ; Jeanneaux et PerrierCornet, 2008). Le concept de conflit d'usage est donc fondamental pour comprendre la répartition du pouvoir entre ruraux, entre autres les polémiques avec les décideurs, surtout les élus municipaux. Ces derniers font l'objet de pressions politiques continues, puisque la stratégie des divers protagonistes est de les convaincre de la légitimité de leur propre argumentation sur les usages. On assiste alors à de vives compétitions entre ces groupes aux conceptions divergentes de la campagne et des zones naturelles, afin que les résolutions municipales soient votées en leur faveur.

Il en résulte une redistribution spatiale des usages qui, loin d'être neutre, témoigne de négociations et de luttes de pouvoir inégales. Les statuts socioprofessionnels et économiques de certains acteurs viennent en effet influencer l'issue de ces hostilités par la mobilisation de leurs expertises, réseaux et capitaux diversifiés (Gravari-Barbas et Veschambre, 2004). Ce qui nous renvoie aux capitaux économiques, culturels, sociaux et symboliques détenus par certains individus privilégiés, au sens de Bourdieu. Peut-on alors penser à une nouvelle forme de capital spécifique à l'environnement? Et quel serait le rôle de ce capital environnemental dans ces rapports de forces inégaux?

\section{Le capital environnemental comme nouvelle clef de lecture}

\section{Complexification des enjeux environnementaux au $\mathrm{XIX}^{e}$ siècle}

L'élaboration d'un nouveau capital sur le modèle des capitaux de Bourdieu est une entreprise audacieuse et risquée. Les débats sur l'inflation des capitaux en sciences sociales en témoignent (par ex : capital spatial, capital scientifique, capital érotique, capital émotionnel...), des auteurs critiquant entre autres leur pertinence et leur autonomie à l'égard des capitaux fondateurs bourdieusiens (notamment Ripoll, 2013; Neveu, 2013; Veschambre, 2006). Pour eux, les capitaux définis par Bourdieu recouvrent l'ensemble de la réalité sociale et rendent inutile l'apparition de capitaux supplémentaires qui 
n'en sont que des dérivés ou combinatoires. À l'inverse, pour d'autres il est possible d'extrapoler de nouveaux capitaux à la faveur de l'évolution inéluctable de la société depuis le tournant du XXI ${ }^{\mathrm{e}}$ siècle, avec notamment les innovations technologiques et les énergies vertes.

En effet, depuis le travail de théorisation de Bourdieu élaboré dans la seconde moitié du siècle dernier, de profondes transformations sont survenues à l'échelle tant nationale qu'internationale. Parmi ces mutations, la complexification des enjeux environnementaux s'impose comme une priorité mondiale incontournable, exigeant de préserver des écosystèmes sains pour les générations futures (PNUE, 2012). Aggravation des changements climatiques, élévation du niveau des mers, destruction de la couche d'ozone, érosion de la biodiversité, raréfaction des ressources telles l'eau et le pétrole, désertification, pollution et diffusion des produits chimiques et des déchets toxiques... sont quelques-uns des événements exigeant la protection urgente des multiples ressources et êtres vivants de la planète.

Le Québec, face à l'activisme des mouvements de protection environnementale, a confirmé son engagement envers notamment le développement durable, l'adaptation aux changements climatiques, la réduction de la dépendance au pétrole, la gestion globale de l'eau y compris la santé des lacs et l'économie de l'eau potable, ainsi que la conservation de la vie sauvage et de son habitat (RNCREQ, 2013). C'est ce contexte environnemental préoccupant et exacerbé par de fréquents conflits qui nous a incitée à revisiter la sociologie bourdieusienne et à « réveiller certains usages ensommeillés de ses concepts, oser poser certaines questions... et développer son imagination sociologique » comme le suggère Lahire (2001a), afin d'y repérer des pistes d'analyse pour mieux saisir les rapports de force environnementaux des divers acteurs ruraux québécois.

\section{Champ environnemental et distribution inégale du capital environnemental}

Pour Bourdieu, le travail du sociologue est de dévoiler les mécanismes de domination et de reproduction des hiérarchies sociales dans la société (Bourdieu et al., 1964, 1967). Cette dernière est analysée comme un univers de différenciations, entre autres selon les classes sociales, les styles de vie, l'accès à des capitaux (Bourdieu, 1979; Lahire, 2001a). Bourdieu scrute donc les rapports sociaux qui sont à l'origine des inégalités et propose une théorie des capitaux. Pour lui, le capital consiste en des ressources qui peuvent être mobilisées et investies, en fonction d'intérêts et d'objectifs précis, par les groupes sociaux dans les différents champs rattachés aux sphères d'activités (culturelles, économiques, sociales, politiques, juridiques, scientifiques, religieux, etc.). Ces champs, microcosmes relativement homogènes et autonomes, sont régis par des lois et codes propres à chacune des sphères. Espaces de domination, ces champs sont composés d'individus qui participent à des luttes sociales pour défendre leurs valeurs et visions, ce qui les positionne les uns par rapport aux autres. Véritables lieux de classement entre dominants et dominés, ces champs sont non seulement le théâtre de concurrences et d'affrontements dans le monde social pour l'appropriation et/ou la redéfinition du capital spécifique au champ, mais aussi un site où se joue une distribution inégale du capital (Bourdieu, 2000 ; Cabin, 2008; Lahire, 2001b). Cette théorie des divers champs sociaux demeure inachevée, Bourdieu n'ayant pu terminer avant sa mort en 2002 une synthèse de ses travaux sur le sujet dans son ouvrage en chantier Microcosmes.

Compte tenu des nombreuses mutations sociétales actuelles et de la prégnance des enjeux et mouvements environnementaux à l'échelle mondiale, nous postulons qu'il est possible d'envisager l'émergence d'un nouveau champ environnemental avec ses propres règles, valeurs spécifiques, enjeux et conflits entre acteurs variés. Car en dépit de frontières poreuses et non complètement fermées entre les divers champs, ces valeurs et luttes environnementales ne sont pas globalement considérées ni intégrées dans les autres champs bourdieusiens. Ce constat justifierait la structuration autonome de ce champ qui est un « champ de forces » à l'instar de l'approche de Bourdieu. Il se révèle, à l'analyse, comme un « champ de bataille » où l'environnement et la nature n'échappent pas aux rapports sociaux de domination, avec comme arrière-scène la forte capacité de résilience du capitalisme (Keucheyan, 2014).

Bourdieu a identifié trois principaux capitaux liés aux champs correspondants. L'existence de ces champs est une condition préalable à toutes formes 
de capitaux. Ces derniers témoignent des asymétries de pouvoir : le capital économique (fortune, salaires, revenus, patrimoine); le capital culturel (savoirs, savoir-faire et savoir-être, référant aux connaissances, diplômes, compétences, valeurs, goûts, biens et titres culturels utiles); le capital social (étendue des réseaux durables de relations et leurs ressources actuelles et potentielles), auquel s'ajoute le capital symbolique (notoriété, prestige, autorité, reconnaissance attribuée par la possession d'autres capitaux). À la différence de Marx, la position sociale d'un individu est moins assignée par son capital économique que par son capital culturel et social, et par la façon dont il les investit. Bourdieu renvoie aux notions de "distinction » qui est un des moteurs des goûts et pratiques sociales pour cultiver sa différence, et de l'« habitus », gestes et manières d'être et de penser acquis inconsciemment depuis l'enfance (par l'école, la famille, les média...) comme normes et marqueurs de l'origine sociale.

S'inspirant de la théorie des capitaux de Bourdieu, notre hypothèse de l'avènement d'un capital environnemental repose sur l'ensemble des investissements (sociaux, économiques, idéologiques, politiques, culturels...) réalisés par divers acteurs dans des biens, connaissances, pratiques, savoir-faire et savoir-être liés à l'environnement, selon leurs intérêts et leur représentations et valeurs environnementales souvent antinomiques (Richard et al., 2017). Ce concept de capital environnemental complète ainsi les études précédentes sur les conflits d'usage en insistant sur les investissements différenciés des acteurs dans ce capital. Puisque le capital environnemental est inégalement réparti parmi les acteurs, l'accès à l'environnement sera restrictif et même impossible pour ceux qui sont pauvres en capitaux. Car Bourdieu (2000), en formulant sa théorie, a clairement associé ses capitaux à des relations empreintes d'iniquités et de discriminations entre individus. Le capital environnemental s'inscrit donc dans un champ autonome environnemental où des acteurs s'affrontent à propos de valeurs environnementales qu'ils priorisent ou non (protection des paysages, potentiel productif de l'environnement, aménités variées, cadre de vie paisible, etc.). Ce capital est alors arrimé à un jeu social complexe où des conceptions et pratiques concurrentes de l'environnement occasionnent des conflits (Richard et al., 2017). Ces conceptions divergentes sont nom- breuses et renvoient aux quatre usages du territoire rural examinés précédemment, par exemple à la vision écologique vs celle commerciale du tourisme rural.

\section{Conversion des autres capitaux et débordement dans l'arène politique}

Pour posséder du capital environnemental, les acteurs doivent opérer une conversion de la totalité ou d'une partie de leurs actifs. Ils investissent alors dans l'environnement en convertissant leurs capitaux préexistants en capital environnemental, en accord avec leurs valeurs environnementales. Par exemple, ils utiliseront leur capital économique pour acheter une résidence dans un endroit où se trouvent des qualités environnementales valorisées (paysages naturels, eau et air purs, sentiers pédestres, espaces protégés, etc.), enrichissant de ce fait leur capital environnemental. Cette conversion en capital environnemental dépend donc de la dimension des autres capitaux détenus, à savoir culturel, économique, social et/ou symbolique. La capacité d'investissement dans le capital environnemental est alors tributaire de l'importance de ces autres capitaux déjà accumulés. Cela entraine l'exclusion pour les personnes sans capitaux, incapables de convertir des actifs pour enrichir leur capital environnemental (Richard et al., 2018). Notons que ces opérations de conversion entre le capital environnemental et les autres capitaux sont réversibles, i.e. le capital environnemental peut être reconverti en capital économique, par exemple par la vente d'une maison située dans un espace protégé.

Or, les enjeux environnementaux ne sont pas les seuls concernés, car les luttes de pouvoir vont s'élucider aussi par les enjeux politiques. Les acteurs ne peuvent éviter d'engager leurs ressources dans l'arène politique, parce que c'est là où se débattent et se ratifient les décisions majeures pouvant influencer le dossier environnemental, tels la planification et l'aménagement territorial. Les deux enjeux sont étroitement liés et les acteurs doivent, pendant leur mobilisation du capital environnemental, élargir leur périmètre d'action pour solliciter l'appui des instances décisionnelles locales. Ils réclament alors des législations et réglementations qui confortent leurs intérêts et représentations de l'espace naturel. Nous postulons que le concept de capital environ- 
nemental offre la possibilité de décrypter ces tractations, stratégies d'influence et rapports de force qui sont au cœur des deux enjeux interreliés. L'un ne se comprend pas sans l'autre pour les pratiques, iniquités et jeux de pouvoir touchant l'environnement. Pour s'assurer d'une victoire lors des contentieux sur l'enjeu politique, les divers acteurs n'hésiteront pas, ici aussi, à enrichir leur capital environnemental de leurs autres ressources liées aux capitaux bourdieusiens, car ce sont des atouts essentiels pour le contrôle de l'environnement et de son développement. Le concept de capital environnemental étant encore en gestation et en discussion, nous allons tester son potentiel avec notre étude de cas au Québec sur le rapport des divers ruraux à l'environnement.

\section{MÉthodologie}

Les données proviennent d'une vaste étude sur les néoruraux au Québec qui explore depuis une dizaine d'années plusieurs thématiques quant à leur intégration en milieu rural (Simard et Guimond, 2009). Elles renvoient précisément ici à un corpus d'entrevues qualitatives permettant de comparer les relations à l'environnement de quatre groupes d'acteurs : les néoruraux, ruraux de longue date, dirigeants d'organismes locaux et élus municipaux. D'une durée moyenne d'une heure et demie, ces entrevues furent menées dans deux territoires ruraux contrastés connaissant l'arrivée de néoruraux au sud du Québec : 1) la $\mathrm{MRC}^{3}$ de BromeMissisquoi, région viticole et touristique reconnue pour ses paysages montagneux et sa vie artistique et où s'installent surtout des retraités; 2) la MRC d'Arthabaska, région agricole et industrielle qui attire une population active et plus jeune (figure 1).

Quatre groupes d'acteurs répartis également dans chacune des MRC furent interrogés. Ils totalisent 93 personnes : 47 néoruraux; 24 ruraux de longue date; 12 dirigeants d'organismes ou d'associations locales; 10 élus municipanx. Les dirigeants et élus forment la catégorie des «décideurs» (tableau 1). Le double statut de ces décideurs, à savoir d'abord comme décideur et ensuite comme néorural ou

3. Il s'agit d'une municipalité régionale de comté (MRC). Une MRC regroupe toutes les municipalités d'un même territoire d'appartenance.

Elle a notamment le pouvoir d'élaborer un schéma d'aménagement pour son territoire et un plan de développement local et régional. On dénombre en 2016 au Québec 87 MRC et 14 villes et agglomérations exerçant certaines compétences de MRC, et un total de 1110 municipalités locales. rural de longue date, a été considéré dans l'analyse et dans le tableau. Notons que les élus ont un profil plus éloigné des autres acteurs : plus âgés (+ de 40 ans), uniquement des hommes et près des trois quarts sont des ruraux de longue date nés dans le territoire de la MRC (7 sur 10). La moitié n’a jamais quitté leur MRC, contrairement aux autres acteurs qui ont massivement séjourné ailleurs, parfois à l'étranger ou dans une autre province canadienne.

Par contraste, les dirigeants sont plus jeunes et se distinguent par le genre (58\% de femmes) et leur double statut mieux distribué que les élus entre les 2 possibilités : à la fois dirigeant et rural de longue date $(7$ sur 12 , i.e. $58 \%)$ ou à la fois dirigeant et néorural ( 5 sur 12 , i.e. $42 \%$ ). Ce double statut plus équilibré des dirigeants peut expliquer leur compréhension plus nuancée des enjeux puisqu'ils ont surement une meilleure connaissance générale des problématiques associées à la néoruralité.

Pour la scolarisation, les néoruraux et les dirigeants sont les plus éduqués avec majoritairement des diplômes universitaires. Ils semblent bien dotés des quatre types de capitaux bourdieusiens qu'ils pourront investir lors des conflits environnementaux. Les élus municipaux, tous des maires, sont les moins scolarisés avec les ruraux de longue date car seulement le quart de ces deux groupes ont des diplômes universitaires. Leurs deux capitaux, culturel et social, risquent d'être plus faibles globalement.

Soulignons que le principal motif de migration des néoruraux relève des qualités environnementales tant physiques que sociales du territoire convoité : paysages champêtres, nature sauvage, air pur, vastes espaces, proximité des loisirs de plein air, cadre de vie sécuritaire pour élever une famille ou prendre sa retraite, convivialité, vie communautaire. Il surpasse de loin les autres motifs qui demeurent minoritaires : professionnels, familiaux, financiers. Ces qualités appréciées, notamment par les néoruraux, indiquent les valeurs essentielles pour lesquelles ils militeront afin d'en assurer la pérennité. Elles seront au cœur des mobilisations de leur capital environnemental pour que prédomine leur vision de la campagne.

L'analyse porte d'abord sur les enjeux environnementaux perçus par les quatre groupes. Pour mieux en cerner la dynamique globale socio-environnementale, les enjeux politiques furent aussi explorés car s'y jouent des rapports de domination 


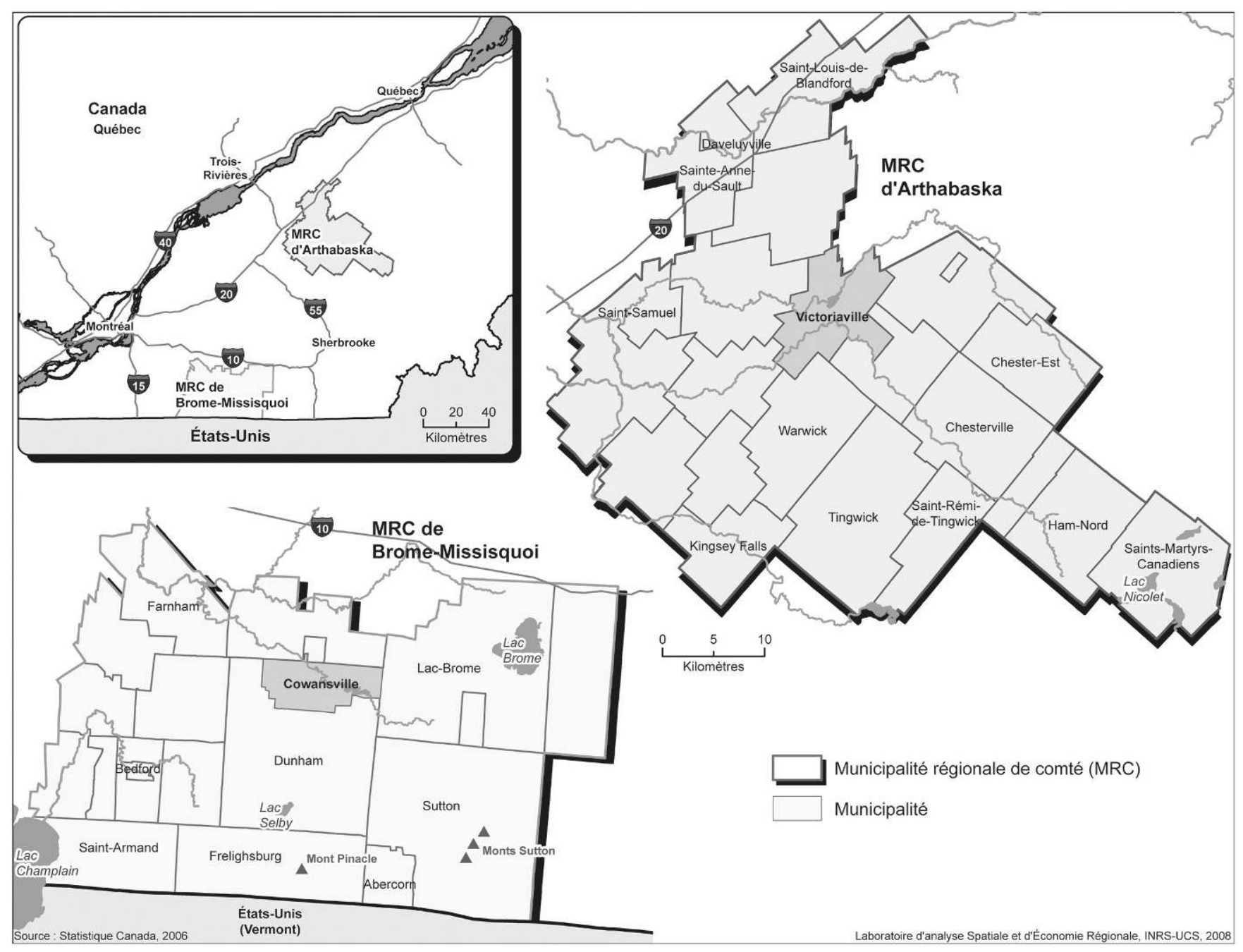

Figure 1 : Localisation des deux MRC à l'étude Location of the two RCMs studied

significatifs en lien avec le capital environnemental. Les visions, valeurs, intérêts, jeux d'alliance ou de concurrence, stratégies et ressources déployées sur ces deux sujets furent examinés. La comparaison des réponses a facilité le repérage tant des convergences, divergences et possibilités d'alliances entre eux, que leurs rapports de pouvoir et mobilisations du capital environnemental.

La parole a été donnée aux acteurs pour en tirer les processus et les logiques, en s'inspirant de la théorie ancrée de Glaser et Strauss (2009). Caractérisée par une démarche de théorisation empirique et inductive pour dégager le sens des événements, elle est ancrée car construite et confirmée par une comparaison continuelle entre la réalité observée et l'analyse qui s'élabore.

\section{LA MOBILISATION DU CAPITAL ENVIRONNEMENTAL DANS LE QUÉBEC RURAL}

Les inégalités de pouvoir et la mobilisation concrète du capital environnemental sont dégagées par l'analyse des enjeux d'abord environnementaux et ensuite politiques dans les deux MRC. Pour faciliter la lecture de ces enjeux, nos données sont présentées toujours dans le même ordre : en premier les expériences et représentations des néoruraux, suivies de celles des décideurs pour terminer par celles des ruraux de longue date qui figurent comme groupe-témoin. 


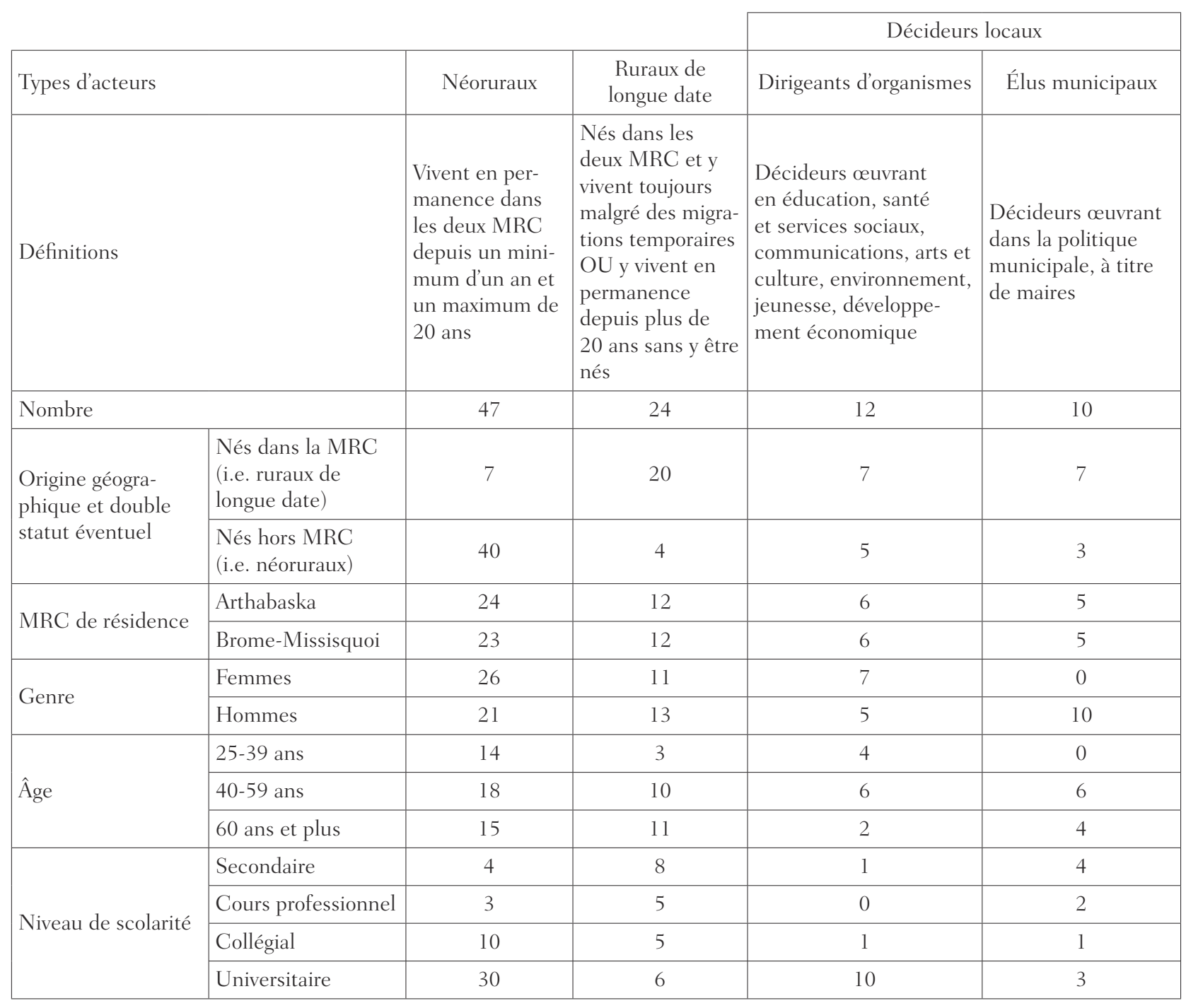

Tableau 1 : Définitions et principales caractéristiques des quatre groupes d'acteurs Definition and main characteristics of the four groups of actors

\section{Les enjeux environnementaux}

Trois enjeux ressortent des entrevues des néoruraux des deux MRC : 1) la protection de l'environnement et la sensibilisation; 2) la réduction de la pollution; 3) la préservation des paysages. Ils sont particulièrement volubiles sur le premier, en spécifiant les dossiers de protection des sites fragiles (étangs, boisés, montagnes), les législations et règlementations complexes à gérer.

Certains néobromisquois préconisent le développement durable, tout en déplorant les difficultés de bloquer les intérêts opposés. Ils rappellent l’impératif primordial d'un équilibre entre le développement économique, social et environnemental et leur res- ponsabilité de chien de garde des élus : «L'enjeu local, ça va être de gérer le développement pour pas qu'il soit sauvage [...]. C'est là qu'on est chien de garde, car l'environnement est un point faible: ils reviennent vite aux anciennes habitudes de mettre $d u$ béton partout » (BMNEO3) ${ }^{4}$. Cette vision exigeant les trois dimensions du développement durable trouve écho dans la littérature, étant estimée indispensable pour la sauvegarde des richesses locales, la revitalisation rurale et la cohésion sociale entre les divers protagonistes (Roy et al., 2005).

\footnotetext{
4. Ces codes identifient les entrevues : $\mathrm{BM}=$ Brome-Missisquoi; $\mathrm{AR}=$ Arthabaska; $\mathrm{NÉO}=$ néorural $; \mathrm{LD}=$ rural de longue date $; \mathrm{DO}=$ dirigeant d'organisme; EM = élu municipal.
} 
Concernant le deuxième enjeu relatif à la pollution, les néoruraux évoquent les algues, la contamination par les produits agricoles, la dégradation des systèmes d'égout et d'aqueduc. Ils réclament une vigilance accrue pour empêcher la détérioration des écosystèmes aquatiques et terrestres et une pénurie d'eau potable. Cette préoccupation témoigne des fréquents conflits d'usage au sujet de cette ressource aussi bien au Québec qui détient 3\% des réserves mondiales d'eau douce, qu'à l'étranger (Manceron, 2006).

Pour le dernier enjeu, les nouveaux résidents dénoncent les répercussions d'une approche «non écologiste » sur les paysages. Combinées aux deux premiers enjeux, elles sont pour eux désastreuses en anéantissant le cachet villageois et son patrimoine naturel et historique (figure 2) : "Pour le paysage, pour le développement, il y a la mairie qui veut telle chose, il y a les résidents qui veulent autre chose. Parce que le haut de la montagne a été trop construit. [...] Vous allez voir les grands condos ${ }^{5}$, un village fantôme sauf à l'époque du ski »(BMNEO4).

Ces résultats concordent avec des études internationales qui signalent cette sensibilité, non exclusive aux néoruraux, envers les dimensions qualitatives et écologiques paysagères ainsi que les conflits et empreintes territoriales dommageables d'un développement mal planifié (Barraud et Portal, 2013; Gobster et al., 2004; Jamal et Eyre, 2003). Plusieurs facteurs, tant économiques que politiques, sociaux, culturels et historiques, élucident les hostilités et les logiques contradictoires de projets concurrents (ex : patrimonialisation vs lotissement).

Certains néoruraux sont conscients qu'ils encouragent l'embourgeoisement rural suite à leur migration, par leurs valeurs, habitudes de vie et mobilisations de diverses ressources dans le capital environnemental. Ceci renvoie à leurs réseaux et revenus pour acheter une propriété près d'une zone d'intérêt écologique, ou à leurs goûts marqués pour des produits de consommation de qualité dont le prix est prohibitif pour les autres villageois (viandes sans hormones, fruits et légumes biologiques, artisanat local, matériaux naturels et recyclables). Les baby-boomers ${ }^{6}$ retraités avec des fonds de pension

5. Condominiums, i.e. immeubles en copropriété au Québec (appartement, logement, maison, bureau).

6. Le baby-boom au Canada s'est étalé sur une période de 20 ans (1946 à 1965). Plusieurs baby-boomers ont déjà atteint 65 ans et sont à la retraite. Parmi cette génération au Québec, figurent des néoruraux retraités ou

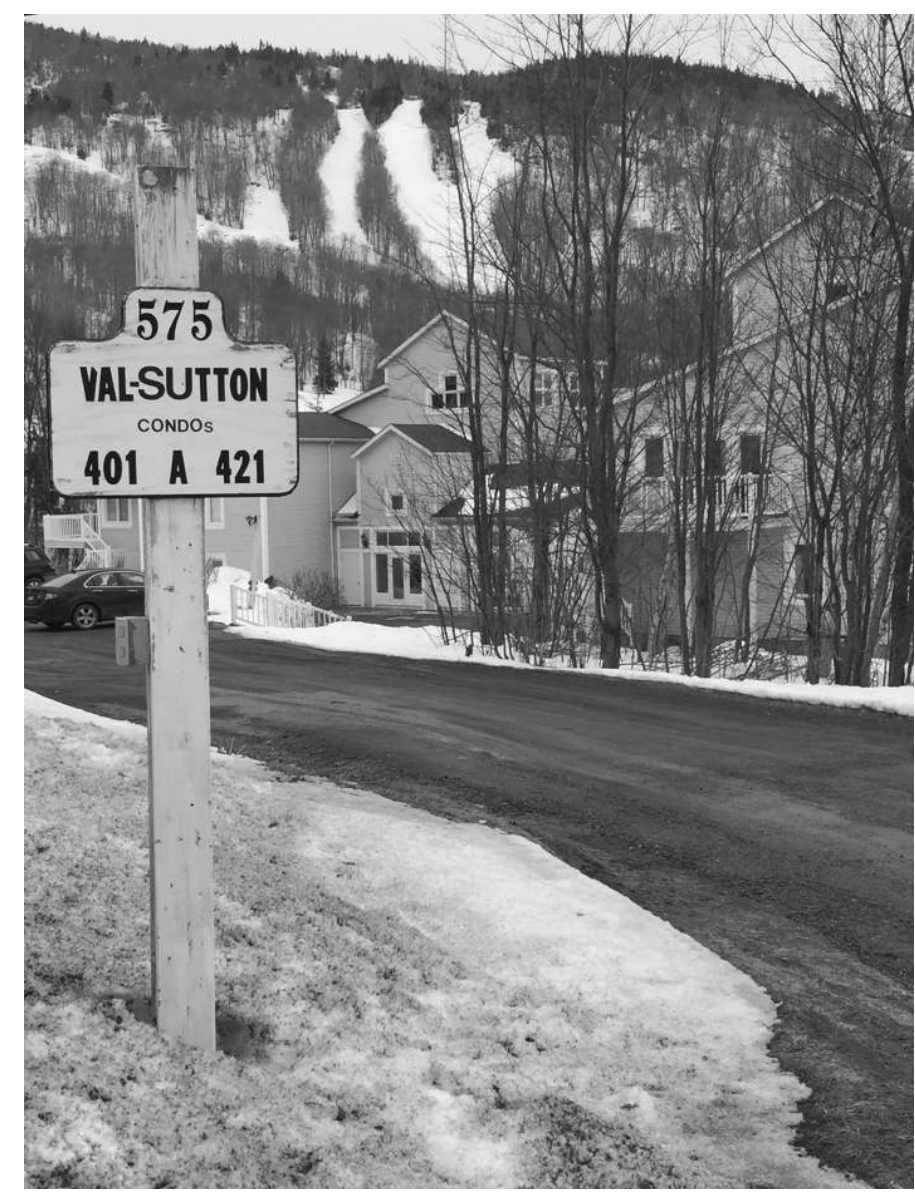

Figure 2 : Condominiums dans les montagnes de Brome-Missisquoi Condominium units in the Brome-Missisquoi mountains

convenables ne sont pas les seuls concernés par ces façons d'investir, de produire et de consommer l'environnement, car d'autres néoruraux y participent par leur quête de vie de qualité, alternative, saine et proche d'une nature préservée et sauvage. La configuration exacte de ces modalités chez un néorural variera selon l'étape de sa vie, un retraité ayant à sa disposition plus de ressources et capitaux qu'un jeune au moment de fonder une famille ou qu'un adulte qui se réoriente au mitan de sa vie. En utilisant ces divers actifs pour s'installer à la campagne, et en convertissant leurs autres capitaux en capital environnemental, les « gentrifieurs » créent une iniquité comme des auteurs l'ont indiqué (Michels, 2017).

D'ailleurs, des chercheurs soulignent les incidences négatives de ces « habitus » de néoruraux qui les distinguent souvent des autres ruraux,

préretraités qui investissent la campagne pour s’y établir définitivement, avec des capitaux diversifiés et avantageux au sens de Bourdieu. 
puisque tant la mobilisation de leurs ressources économiques, sociales et culturelles que leurs pratiques (rénovation, jardins bios, achats de produits raffinés...) entrainent un embourgeoisement ou une « greentrification » des campagnes où les valeurs environnementales ont joué un rôle déterminant dans leur migration rurale (Richard et al., 2014; Smith et Phillips, 2001). Cette avidité des nouveaux résidents pour les espaces verts occasionne une augmentation de la valeur foncière, l'exclusion des moins nantis, le remplacement des commerces locaux par d'autres plus luxueux et un sentiment de dépossession des ruraux de longue date: "Les jeunes ne peuvent pas rester ici, les maisons ne sont plus achetables » dénonce l'un d'eux (BMLD35). Cela attise l'animosité envers les nouveaux arrivants fortunés et « gentrifieurs » (Guimond et Simard, 2010 ; SolanaSolana, 2010). En s'appropriant ainsi symboliquement et matériellement des territoires ruraux à forte aménité environnementale, ces néoruraux protègent à la fois les qualités de leur habitat et leur reproduction sociale et culturelle, tout en marginalisant des populations à statuts socio-économiques précaires, incapables d'investir dans le capital environnemental et de prescrire leurs visions (Phillips, 1993). Ceci touche surtout Brome-Missisquoi où les constructions de condos et de maisons de luxe en montagne nuisent à l'abordabilité de l'habitation : «Les taxes ont flambé. C'est cool de rentrer des taxes..., mais si les gens de la place sont obligés de s'en aller parce qu'ils ne sont plus capables de les payer, bien ça c'est quelque chose de majeur » (BMDO9).

Qu'en est-il des autres acteurs? À part quelques exceptions dans Brome-Missisquoi, les décideurs et ruraux de longue date n'adhèrent nullement aux multiples enjeux des néoruraux. Personne, en effet, ne soulève la préservation des paysages, car c'est un sujet sensible en raison des projets décidés en catimini et qui ont détruit des paysages locaux, tels les coupes à blanc de certaines montagnes. Ayant tous une appréciation partielle, les élus et ruraux de longue date ne considèrent que la pollution, et les dirigeants uniquement la protection et sensibilisation environnementales. Ils expriment ainsi des conceptions sur l'usage des espaces naturels qui sont contraires aux visions des néoruraux. Ceci engendre des rapports de forces surtout entre des néoruraux qui s'imposent comme défenseurs écologiques et des maires qui semblent appuyer plus les contracteurs et promoteurs de projets, tels les lotissements, pour les rentrées de taxes.

Cette position des maires, davantage pour le développement que pour la conservation du territoire, rejoint d'autres analyses en France où les causes les plus fréquentes des conflits sont la remise en question de la protection des milieux ruraux et littoraux par des pratiques préjudiciables d'urbanisme et de promotion immobilière entérinées par des élus (Cadoret et al., 2012). Au Québec, pour justifier leur choix de développement économique, ces derniers accusent les néoruraux d'être responsables des conflits d'usage avec leur représentation trop idyllique (Simard, 2007). Avec quelques dirigeants, ils s'insurgent contre cette vision néorurale stéréotypée : "[C'est] une campagne typique d'une carte postale où la réalité est déformée et simpliste, par exemple sans mouches, bruits de grenouilles, boues et manvaises routes... (BMDO8).

Une réduction des tensions à une opposition duale entre nouveaux résidents et ruraux de longue date serait cependant abusive. La situation est plus complexe car des collaborations existent au Québec lors d'engagements citoyens pour la protection de l'environnement (Guimond et Simard, 2011). Par exemple, une mobilisation, organisée à partir de 2006, par des citoyens du Groupe Sawvons les Cantons pour lutter contre un projet de carrière en zone agricole dans Brome-Missisquoi, regroupait tant néoruraux que ruraux de longue date des quatre villages touchés par ce projet (Sutton, Frelighsburg, Dunham, Abercorn). Menaçant l'environnement et le patrimoine paysager et floristique de la région, ce projet était illégal par sa non-conformité avec la Loi sur la protection du territoire et des activités agricoles du Québec et le règlement de zonage municipal. Parmi leurs actions, une pétition fut lancée en 2011, destinée à la Commission de Protection du Territoire Agricole du Québec (CPTAQ), pour contester le renouvellement de cette « carrière agricole » pour trois ans. Le Groupe recueillit sans peine plus d'une centaine de signatures de nouveaux et d'anciens résidents en seulement deux jours, illustrant la complicité et l'entraide entre ces deux groupes (figure 3).

En somme, les investissements dans le capital environnemental des ruraux des deux MRC sont disparates, à l'instar de leurs conceptions et valeurs différenciées de l'environnement. Les néoruraux sont ceux qui semblent mobiliser davantage l'ensemble 


\section{PÉTITION CONTRE LA GRAVIÈRE-AGRICOLE D'ABERCORN ! NON AUX PSEUDO-PROJETS AGRICOLES !}

\section{ATTENDU...}

- Que depuis 3 ans la Commission de Protection des Territoires Agricoles (la CPTAQ) autorise un promoteur d'Abercorn à faire des activités de nivellement, d'extraction et de vente de sable et de gravier ;

- Qu'après trois années de travaux, le site en question ressemble à un terrain bombardé et n'est toujours pas nivelé, alors que le réaménagement et l'ensemencement devaient se faire un demi-hectare à la fois, tel qu'exigé dans la décision de la CPTAQ de 2006;

- Qu'il y a eu plutôt «EXPLOITATION COMPLĖTEMENT DÉSORDONNÉEx selon les termes mêmes de la CPTAQ, et qu'on s'est contenté de CREUSER, d' ENLEVER et de VENDRE du sable et du gravier ;

- Que le promoteur a vendu du sol arabe (constaté dans une mise en demeure de la CPTAQ) alors que cette terre doit être conservée pour le retour du site à des fins agricoles :

- Que le lieu dans lequel se trouve la «Gravière-Agricole» est un secteur de villégiature zoné «Récrég-Forestier» et NON une zone d'opération et d'exploitation de gravière ;

- Que le promoteur a fait plusieurs infractions constatées autant par la CPTAQ que par le Ministère du Développement Durable, de r'Environnement et des Parcs (le MDDEP);

- Qu'une nouvelle demande faite par le même promoteur est l'objet d̛une orientation préliminaire de la CPTAQ autorisant: $\quad-3$ ans supplémentaires de travaux

- le doublement de la surface à niveler

- le déboisement d'un hectare de forêt et le reboisement d'un hectare de champ plat.

NOUS SOUS-SIGNEES CONSIDEERONS QUE CE PROJET NE CONSTITUE PAS UN PROJET D'AMĖLIORATION AGRICOLE ET DEMANDONS A LA COMMISSION DE PROTECTION DES TERRITOIRES AGRICOLES...

- De refuser toute demande actuelle ou future concernant le nivellement de ce site ;

- De cesser de servir de paravent à des activités qui n’ont d’agricoles que le nom ;

- De cesser d'encourager, sous prétexte d'agriculture, le développement de gravières et sablières dans une région qui en compte déjà près de $\mathbf{4 0}$;

- De reconnaître que la région concernée par cette «Gravière-Agricole» est une région de montagnes et de collines et non une plaine agricole ;

- De reconnaître qu'un projet dit agricole prétendant transformer des collines en prairies ne peut être rentable dans les conditions actuelles de notre économie.

Signatures : Noms, Adresses

Groupe Sauvons les Cantons (3-2011)

Figure 3 : Pétition « Sauvons nos cantons »

"Save our townships" petition

de leurs ressources et convertir leurs autres capitaux en capital environnemental afin d'imposer leurs visions, parfois appuyés par des ruraux de longue date et des dirigeants ${ }^{7}$. Mais sur plusieurs dossiers, ils ont à confronter les maires qui banalisent souvent

7. On observe un comportement variable pour les ruraux de longue date, intervenant tantôt comme alliés, tantôt comme opposants des nouveaux leurs revendications. Or, la compréhension des pratiques environnementales contrastées et inégales est incomplète. 11 faut chercher ailleurs d'autres clefs d'interprétation. Les enjeux politiques apportent un éclairage complémentaire.

résidents. Quant aux dirigeants, ils sont plus enclins aux compromis vu une meilleure appréhension globale des enjeux que les élus. 


\section{Et derrière... les enjeux politiques}

Les enjeux politiques, parce qu'ils s'inscrivent au cœur des conflits d'usage et des visions rivales du développement, canalisent des interactions insatisfaisantes des quatre groupes à propos de deux sujets sensibles : 1) la gestion des municipalités;2) leur aménagement territorial. Ces sujets expliquent les conflits soulevés par des investissements contradictoires dans le capital environnemental. Alors que les néoruraux des MRC étudiées identifient ces deux sujets, les autres acteurs se limitent au premier, en ignorant l'aménagement pourtant fondamental parmi les responsabilités municipales.

Un consensus semble d'abord se dégager dans les deux territoires concernant la gestion municipale, mais un écart ressort rapidement avec les élus. Pour les dirigeants et les ruraux, anciens et nouveaux, il s'agit surtout d'assurer une saine gouvernance par davantage de transparence et d'ouverture et une meilleure participation citoyenne dans les décisions : "Les conseillers municipaux, il y en a qui sont ouverts, il y en a d'autres qui trouvent donc fatigant quand le néorural veut avoir tel service » (ARNÉO6). En revanche, les maires soulèvent d'autres aspects de leur gestion, spécialement les coûts élevés des services et l'insuffisance des finances. Certains critiquent les demandes déraisonnables et « urbaines » des nouveaux ruraux, tels le transport collectif et leur insistance sur la règlementation et la consultation. Ces élus paraissent moins réceptifs à leurs doléances, les considérant parfois comme des «pacotilles».

Ces controverses entre néoruraux et élus sur l'administration municipale ont déjà été observées (Bossuet et Simard, 2013). Elles visent tant l'agacement des élus devant des requêtes coûteuses, que les frustrations des nouveaux résidents envers leur exclusion et les décisions précipitées : «Le budget a été revoté, mais ils ont fait ça un 22 décembre à 19 hrs le soir. J'étais dans les préparatifs de Noël et je n'y suis pas allé » (ARNÉO11). Une fracture manifestant une perte de confiance mutuelle apparaît : d'un côté, des néoruraux refusent certaines visions et résolutions municipales jugées inéquitables, illégales et incohérentes, et proposent des alternatives. De l'autre, des élus envisagent habituellement ces démarches comme des perturbations et une menace à leur capacité de gérer le bien public, plutôt qu'une occasion d'échanges pour convenir d'objectifs communs. D'où la marginalisation des néoruraux et autres opposants pour préserver leur autorité. Déjà en 2000, Kayser présageait ces luttes de pouvoir entre les nouveaux venus « éventuellement candidats à des responsabilités municipales » et « un pouvoir notabilaire [sic] qui s'effrite » (p. 103).

Seuls les néoruraux des deux MRC ajoutent le deuxième enjeu de l'aménagement territorial par les municipalités, crucial pour la préservation des qualités paysagères et environnementales des villages et la planification future du développement. Inquiets du favoritisme et des interventions improvisées, ils tentent de faire redresser ces stratagèmes par davantage de cohérence dans la planification et de respect, par les élus et les promoteurs, du plan d'urbanisme municipal et de son règlement de zonage.

Le mécontentement des néoruraux est palpable et risque de déclencher des frictions et mobilisations, puisque personne d'autre n'aborde ce sujet, y compris les maires, principaux pivots des résolutions névralgiques sur la planification territoriale. Des chercheurs exposent cette conflictualisation de l'aménagement rural : collusions d'intérêts dans l'élaboration et l'application de plans stratégiques, visions discordantes des utilisations du sol, législations imprécises, irrationalités dans l'implantation, intimidations, dissimulation des nuisances. D'autres examinent les mobilisations localisées qui s'opposent aux décisions unilatérales de l'autorité publique et à sa conception étroite de la protection environnementale. Ces conflits sont alors interprétés comme une remise en cause de la légitimité des décideurs et une condamnation des nuisances créées par des projets d'aménagement (Dechézelles et Olive, 2016). La position compliquée de l'élu « entre le marteau et l'enclume » et l'arrivée des néoruraux comme «facteurs de contestation » sont soulignées (Subra, 2006).

Les enjeux politiques attisent donc des tensions entre élus municipaux et néoruraux autant sur des enjeux procéduraux (transparence et consultation) que structurels (légitimité de l'action publique, démocratisation) (Dziedzicki, 2004). Outre cette crise de confiance, le processus décisionnel et les orientations politiques sont contestés pour pouvoir rediscuter de l'intérêt général et co-construire une mixité équilibrée des usages où la préservation environnementale aurait sa place. Devant la quasi-indif- 
férence des élus et leurs pratiques irrationnelles, les néoruraux doivent redoubler d'efforts pour obtenir gain de cause. Avec leurs alliés, ils vont engager toutes leurs ressources disponibles pour consolider leur pouvoir tout en renforçant et enrichissant leur capital environnemental des atouts et possibilités des autres capitaux bourdieusiens. La mobilisation et conversion de leurs capitaux préexistants en capital environnemental permet de mieux les doter pour garantir l'implantation du projet désiré et imposer des valeurs écologiques.

\section{Un exemple : le projet d'une carrière de sable dans Brome-Missisquoi}

Le projet déjà mentionné d'extraction de sable dans un champ agricole dans Brome-Missisquoi illustre la confrontation d'acteurs autour d'un plan compromettant les espaces naturels. Il révèle que le capital environnemental réquisitionné par différents acteurs n'évolue pas en vase clos et est activé sur plusieurs scènes, tant politique que juridique et administrative. Le capital environnemental est alors maximisé par l'apport des autres ressources et capitaux détenus par ces acteurs afin de rallier les décideurs et autres ruraux à leur cause. Cependant se constatent des inégalités quant aux investissements dans l'environnement pour les individus détenant peu de capitaux, puisque leur accès aux réseaux, ressources et experts peut être limité et moins bénéfique comparativement aux acteurs prépondérants et bien pourvus en capitaux.

Ce projet d'une sablière fut rapidement débattu dans les instances politiques locales et régionales. Par un règlement, le conseil municipal du principal village concerné a d'abord donné son accord pour développer cette exploitation en 2006 pour assurément en récolter les bénéfices de taxation. Le comité de citoyens, dénommé le Groupe Sauvons les Cantons au membership métissé de ruraux - nouveaux et anciens - de quatre villages voisins, s'est formé rapidement pour contrer ce projet illégal. Ses membres ont dû s'investir alors au niveau local, en faisant plusieurs interventions à l'hôtel de ville sur les conséquences négatives et les nuisances, telles la pollution d'un cours d'eau et l'insécurité due à la circulation des camions etc. Aussi, ce groupe appuya l'élection d'une conseillère municipale qui s'avéra indispensable comme « chien de garde », spécifique- ment pour décourager les décisions arbitraires et les collusions éventuelles avec des entrepreneurs. Du harcèlement et des menaces anonymes suscitèrent chez les citoyens une méfiance et peur qui entravèrent la quête de compromis. Un combat acharné se prolongea pendant plusieurs années, où les réunions houleuses du conseil municipal opposèrent le Groupe Sauvons les Cantons à plusieurs conseillers et citoyens favorables au projet. Comme chaque groupe tentait d'engager le maximum de ses ressources disponibles dans ces luttes, il en résulta des représentations conflictuelles de l'environnement comportant des valeurs et intérêts incompatibles.

Des actions et des tractations diversifiées furent entreprises au fil des ans par les deux parties rivales, dans plusieurs champs (politique, juridique, administratif) pour valoriser leurs représentations de l'environnement. Certaines donnent le ton de cette lutte teintée d'intimidation : temps limité de parole par le maire pour les opposants pendant les réunions du Conseil, non-respect des règlements notamment celui sur les nuisances, conflits d'intérêt (le propriétaire de la carrière siégeant depuis 2009 comme conseiller), désinformation et difficulté d'accès à certains documents à l'hôtel de ville, etc. Les controverses entrainèrent plusieurs batailles juridiques et administratives. Les capitaux économiques, sociaux, culturels et symboliques furent alors sollicités dans chacun des camps pour payer des experts (agronomes, avocats, enquêteurs), témoigner adéquatement devant les tribunaux, rédiger des déclarations, rapports et lettres pour convaincre les décideurs et politiciens, etc. Tous diversifièrent leurs actions : émissions télévisées, articles dans les journaux ${ }^{8}$, requêtes à la MRC et autres instances gouvernementales, rencontres avec des décideurs locaux et provinciaux, relevés des infractions de la carrière pour les ministères concernés, pétition, etc. Chacun tentait de tirer son épingle du jeu, en utilisant au maximum ses expériences, compétences, notoriétés, contacts, statut social. Furent avantagés lors de ces investissements dans le capital environnemental les individus dotés de connaissances et de savoir-faire utiles, de réseaux étendus (professionnels, politiques, médiatiques...), et des aptitudes pour décoder les informations et élaborer des stratégies globales.

\footnotetext{
8. Voir l'article Les Fraises du journaliste Pierre Foglia dans le quotidien La Presse en 2011 : [http://www.lapresse.ca/debats/chroniques/pierrefoglia/201104/10/01-4388392-les-fraises.php].
} 
Finalement, après une saga de huit ans et maintes péripéties, une résolution municipale vint clore ce dossier en juillet 2014. Le nouveau Conseil municipal refusa en effet d'appuyer une nouvelle demande de renouvellement du permis pour deux ans, compte tenu des infractions passées, de l'absence de nivellement graduel du terrain pour une remise en culture tel qu'exigé par la CPTAQ, et de la non-conformité de cet usage à la réglementation en vigueur. En 2016, le terrain et la maison furent vendus à un nouveau propriétaire qui n'a plus le droit d'exploiter la carrière. Depuis, de nouveaux conflits d'usage ont émergé dans la même $\mathrm{MRC}$, à propos entre autres de la multiplication des antennes de communications cellulaires. On leur reproche de créer de la pollution visuelle dans un paysage montagneux apprécié et d'accélérer le déboisement des collines. Il est à prévoir que d'autres conflits environnementaux vont continuer à survenir, surtout avec l'installation à la campagne d'individus sensibilisés à la qualité environnementale.

Pour terminer, mentionnons que notre analyse des deux MRC et cet exemple d'une carrière de sable mettent bien en évidence des conceptions contradictoires du développement et de la protection de l'environnement qui s'opposent. Celles-ci se dévoilent au fil des investissements contrastés des quatre groupes dans le capital environnemental et des conflits qui en résultent. Elles élucident le fondement et la signification des luttes d'intérêts et de pouvoir des protagonistes mécontents de la cohabitation difficile des divers usages dans leur territoire.

Nous pouvons condenser ces conceptions en deux grandes tendances : d'un côté celle d'un environnement à protéger inscrit dans un plan de développement durable et équilibré. Les porteurs sont surtout les néoruraux et leurs alliés qui privilégient les usages de préservation ou de récréation (ex : parcs naturels, sentiers pédestres, sites historiques patrimoniaux...); de l'autre un environnement au service d'un développement territorial plus intense axé notamment sur la construction de lotissements d'habitations et d'infrastructures de services commerciaux, agricoles... (ex : centres d'achat, tours de communication cellulaire, porcheries...) et d'activités industrielles (ex : carrières, usines...). Les principaux porteurs sont les élus municipaux associés à des promoteurs et entrepreneurs qui favorisent davantage les usages productifs et résidentiels. En somme, ces deux visions préconisent un développement, mais qualitativement et structurellement différent.

\section{Conclusion}

Dans le contexte contemporain où l'environnement est un enjeu planétaire au cœur de contestations régulières, l'émergence du concept de capital environnemental recouvre une réalité indéniable et semble avoir sa pertinence et sa spécificité quant aux capitaux fondateurs bourdieusiens. À la lumière de notre étude de cas québécoise, ce concept apparait utile comme clef de lecture pour appréhender les défis de la crise écologique actuelle ainsi que la dynamique et la complexité des rapports environnementaux socialement différenciés. Il a notamment permis de mieux comprendre les stratégies de domination et de pouvoir/contre-pouvoir souvent occultées des acteurs ainsi que les inégalités lors des négociations environnementales et politiques. De ce fait, il a démystifié le processus de structuration d'un pouvoir hiérarchisé et identifié des conséquences négatives, telles l'exclusion de populations peu nanties en capitaux.

En réponse à l'interrogation du titre de l'article, ce concept a bien mis en relief une mobilisation inégale du capital environnemental parmi les quatre acteurs analysés, les plus actifs étant les néoruraux auxquels font face les élus municipaux. Les deux ont des ressources diversifiées et distinctes, tout en convertissant les autres capitaux qu'ils détiennent en capital environnemental. D'autres acteurs sont moins actifs, faute de capitaux et d'atouts en raison d'un faible niveau de scolarité, de l'absence d'un réseau dense de contacts etc. Loin de nous cependant l'intention de généraliser les constats dégagés pour les deux MRC québécoises à l'ensemble des territoires ruraux de divers pays. Car chaque espace présente des particularités singulières relativement aux acteurs, enjeux, valeurs et hiérarchies sociales que les chercheurs devront identifier et mettre en perspective.

En fait, plusieurs questions subsistent et justifient de poursuivre les discussions sur ce concept encore en construction, car des zones d'ombre sont à clarifier. Ainsi, l'argumentation de l'autonomie de ce capital environnemental vis-à-vis les capitaux de Bourdieu doit être davantage peaufinée, en oppo- 
sition à son incorporation comme simple dimension environnementale de ces capitaux, ou encore comme une espèce ou combinaison particulière du capital culturel ou social. Comment se démarque$\mathrm{t}$-il des autres capitaux avec lesquels il interagit immanquablement? En quoi est-il complémentaire des autres formes de capital dans la sociologie bourdieusienne? Outre le contexte de valorisation de l'environnement, quels sont les autres facteurs qui contribuent activement à sa spécificité comme capital autonome et qui le consolident? Par quels mécanismes de nouveaux capitaux peuvent-ils émerger? Quels en sont les gages de qualité et de pérennité?

D'autres questions peuvent aussi être posées dans la foulée des changements structurels qui apparaissent présentement au XXI ${ }^{\mathrm{e}}$ siècle et qui affectent le rapport de l'homme à l'environnement, telles les méga-exploitations agricoles, les innovations technologiques, les dérèglementations, les changements climatiques, l'économie du savoir, etc. Comment le concept de capital environnemental va-t-il s'ajuster à ces transformations profondes et les interpréter au fur et à mesure de leur évolution? Ce concept traversera-t-il l'épreuve du temps?

Le débat reste donc ouvert sur ce nouveau concept environnemental. Il importe de demeurer à la fois réceptif aux divergences et discussions et attentif aux exigences de rigueur théorique et de clarification conceptuelle, vu la richesse et la complexité de la notion de capital dans ses formes contemporaines.

\section{Remerciements}

Mes remerciements au CRSH, FRQSC et au partenariat Familles en mouvance de l'INRS pour leur appui financier. Un merci spécial à l'équipe du laboratoire GEOLAB UMR 6042 CNRS - université de Limoges où je fus invitée pour un séjour de recherche, et qui a inspiré l'approche de cet article.

\section{Bibliographie}

Abrams J., Gosnell H., Gill N., Klepeis P., 2012. Re-creating the rural, reconstructing nature: An international literature review of the environmental implications of amenity migration, Conservation and Society, n ${ }^{\circ}$ 3, vol. 10, p. 270-284.
Barraud R., Portal C., 2013. Les petites vallées des Pays de la Loire. Du paysage pittoresque aux nouvelles interprétations patrimoniales : permanences et tensions contemporaines, Norois, no 228, vol. 3, p. 11-26.

Bossuet L., Simard M., 2013. Mutations des espaces ruraux sous l'effet des migrations : regards croisés entre la France et le Québec, numéro spécial double, Géographie, Économie, Société, $\mathrm{n}^{\circ}$ 1-2, vol. 15, p. 5-9.

Bourdieu P., 2000. Propos sur le champ politique, Presses universitaires de Lyon, 110 p.

Bourdieu P., 1979. La distinction. Critique sociale du jugement, Paris, Minuit, $672 \mathrm{p}$.

Bourdieu P., Passeron J.-C., 1964. Les héritiers. Les étudiants et la culture, Paris, Minuit, 1964, 192 p.

Bourdieu P., Passeron J.-C., Chamboredon J.-C., 1967. Le métier de sociologue, Paris, Mouton, 432 p.

Boyle P., Halfacree K., 1998. Migration into rural areas: theories and issues, Wiley, England, 338 p.

Brunori G., Rossi A., 2007. Differentiating countryside: social representations and governance patterns in rural areas with high social density: the case of Chianti, Italy, Journal of Rural Studies, n 2, vol. 23, p. 183-205.

Cabin P., 2008, Dans les coulisses de la domination, in Fournier M. (dir.), Pierre Bourdieu, Son œeuvre, son héritage, Seuil, coll. « Sciences Humaines », p. 42-51.

Cadieux K., Hurley P., 2011 . Amenity migration, exurbia, and emerging rural landscapes, Special Issue, GeoJournal, $\mathrm{n}^{\circ} 4$, vol. 76, p. 297-446.

Cadieux K., 2011 . Competing discourses of nature in exurbia, GeoJournal, n 4, vol. 76, p. 341-363.

Cadoret A., Darly S., Kirat T., Lavaud-Letilleul V., 2012. Conflits d'usage littoraux et intérieurs: une géographie du contentieux de seconde instance dans le département de l'Hérault, Norois, no 225, vol. 4, p. 29-46.

Cloke P., Goodwin M., 1993. Regulation, green politics and the rural, in Harper S. (ed) The greening of rural policy: International perspectives, Belhaven, p. 27-41.

Cloke P., Thrift N., 1987. Intra-class Conflict in rural areas, Journal of Rural Studies, n ${ }^{\circ}$ 4, vol. 3, p. 321-333.

Dechézelles S., Olive M., 2016. Lieux familiers, lieux disputés - dynamiques des mobilisations localisées, Introduction aux numéros doubles sur Conflits de lieux, lieux de conflits, Norois, no 238-239, vol. 1-2, p. 7-21.

Dziedzicki J.-M., 2004. Au-delà du Nimby : le conflit d'aménagement, expression de multiples revendications, in Melé et al. (dir.), Conflits et Territoires, Tours, Presses universitaires François-Rabelais, p. 35-64.

Glaser B., Strauss A., 2009. The Discovery of Grounded Theory: Strategies for Qualitative Research, Transaction Publishers, USA, 271 p.

Gobster P.H., Stewart S.I., Bengston D.N., 2004. The Social Aspects of Landscape Change: Protecting Open Space Under the Pressure of Development. Special issues, Landscape and Urban Planning, no 2-3, vol. 69, p. 149-334.

Gosnell H., Abrams J., 2011 . Amenity migration: diverse conceptualizations of drivers, socioeconomic dimensions, and emerging challenges. GeoJournal, no 4, vol. 76, p. 303-322. 
Gravari-Barbas M., Veschambre V., 2004. Patrimoine : derrière l'idée de consensus, les enjeux d'appropriation de l'espace et des conflits, in Melé et al. (dir.), Conflits et Territoires, Tours, Presses universitaires François-Rabelais, p. 67-82.

Guimond L, Simard M., 2011. Les néo-ruraux et les ruraux de longue date sont-ils si différents au Québec? Analyse de leur mobilité, sens des lieux et engagement, Canadian Journal of Regional Science, no 4, vol. 34, p. 151-163.

Guimond L., Simard M., 2010. Gentrification and neo-rural populations in the Québec countryside: Representations of various actors, Journal of Rural Studies, vol. 26, p. 449-464.

Jamal T., Eyre M., 2003. Legitimation Struggles in National Park Spaces: The Banff Bow Valley Round Table. Journal of Environmental Planning and Management, $\mathrm{n}^{\circ}$ 46, vol. 3, p. 417-442.

Jeanneaux P., Perrier-Cornet P., 2008. Les conflits d'usage du cadre de vie dans les espaces ruraux et la décision publique locale. Économie rurale, no 306, p. 39-54.

Kayser B., 2000. L'intégration de la ruralité : les campagnes françaises au $\mathrm{XxI}^{\mathrm{e}}$ siècle. Économie rurale, $\mathrm{n}^{\circ} 255$ 256, p. 100-103.

Keucheyan R., 2014, La Nature est un champ de bataille. Essai d'écologie politique, Paris, La Découverte, coll. «Zones », $208 \mathrm{p}$.

Kirat T., Torre A., 2006. Conflits d'usages et dynamiques spatiales. Les antagonismes dans l'occupation des espaces périurbains et ruraux (I). Géographie, Économie, Société, no 3, vol. 8, p. 293-298.

Lahire B. (dir.), 2001 a, Le travail sociologique de Pierre Bourdieu : dettes et critiques, La Découverte, Paris, 2001, 322 p.

Lahire B., 2001b, Champ, hors-champ, contrechamp, in Le travail sociologique de Pierre Bourdieu: dettes et critiques, Paris, La Découverte, p. 23-57.

LÉvesque E., 2015, Technomades, les expatriés volontaires, Lapresse.ca, Montréal, 25 janvier.

Manceron V., 2006. Discordes territoriales: les logiques rivales de la gestion de l'eau dans une région d'étangs française, Géographie, Économie, Société, no 3, vol. 8, p. 319-328.

Martin N., Bourdeau P., Daller J.-F., 2012. Les migrations d'agrément: du tourisme à l'habiter, Paris, L'harmattan, $412 \mathrm{p}$.

Michels J., 2017. Permanent Weekend, Nature, Leisure, and Rural Gentrification. McGill-Queen's University Press, Montréal, $280 \mathrm{p}$.

Neveu É., 2013. «Les sciences sociales doivent-elles accumuler les capitaux? À propos de Catherine Hakim, Erotic Capital, et de quelques marcottages intempestifs de la notion de capital », Revue française de science politique, $\mathrm{n}^{\circ} 2$, vol. 63, p. 337-358.

Perrier-Cornet P., 2002. À qui appartient l'espace rural? Enjeux publics et politiques, Paris, Éditions de l'Aube/Datar, $141 \mathrm{p}$.

Phillips M., 1993. Rural gentrification and the processes of class colonization. Journal of Rural Studies, $\mathrm{n}^{\circ}$ 9, vol. 2, p. 123-140.

Pistre P., 2010. Mobilisation dans les campagnes toulousaines : conflit d'aménagement et tensions locales. Espaces et sociétés, no 143, p. 151-169.
Programme des Nations unies pour L'environnement (PNUE), 2012, L'avenir de l'environnement mondial, Geo-5, $5^{\mathrm{e}}$ rapport, $54 \mathrm{p}$.

Regroupement National des Conseils régIONAUX DE L'ENVIRonnement du Québec (RNCREQ), 2013, Les grands enjeux 2013, Environnement Québec et régions, 28 p.

Richard F., Saumon G., Tommasi G., 2017. Le capital environnemental, nouvelle clé d'interprétation de la gentrification rurale?, Norois, n 243.

Richard F., Saumon G., Tommasi G., 2018. Des enjeux environnementaux à l'émergence d'un capital environnemental ? Proposition de lecture des inégalités sociales par le prisme de l'environnement, Vertigo (sous presse).

Richard F., Dellier J., Tommasi G., 2014. Migration, environnement et gentrification rurale en Montagne limousine. Revue de géographie alpine, $\mathrm{n}^{\circ} 102$, vol. 3. [En ligne].

Ripoll F., 2013. Quelle dimension spatiale des structures sociales chez Bourdieu? Localisations résidentielles et jeux d'échelles dans La Distinction, in Coulangeon Ph., Duval J. (dir.), Trente ans après La Distinction, Paris, La Découverte, p. 365-377

Roy L., Paquette S., Domon G., 2005. La campagne des néoruraux : motifs de migration, territoires valorisés et usages de l'espace domestique. Recherches sociographiques, vol. XLVI, p. 35-65.

Simard M. (dir.), 2011. Les nouveaux ruraux dans les campagnes au Québec et en France : impacts et défis, numéro spécial. Revue canadienne des sciences régionales, $\mathrm{n}^{\circ} 4$, vol. 34, p. 105-223.

Simard M., 2007. Nouvelles populations rurales et conflits au Québec : regards croisés avec la France et le Royaume-Uni. Géographie, Économie, Société, no 2, vol. 9, p. 187-214.

Simard M., Guimond L., 2009. L'hétérogénéité des nouvelles populations rurales : comparaison dans deux MRC contrastées au Québec. Recherches sociographiques, $\mathrm{n}^{\circ} \mathrm{L}$, vol. 3, p. 475-505.

SleE R.W., 2005. From countrysides of production to countrysides of consumption? The Journal of Agricultural Science, $\mathrm{n}^{\circ} 143$, vol. 4, p. 255-265.

Smith D.P., Phillips D.A. 2001. Socio-cultural representations of greentrified Pennine rurality, Journal of Rural Studies, $\mathrm{n}^{\circ} 17$, p. 457-469.

Smith M.D., Krannich R.S., 2000. "Culture Clash” Revisited: Newcomer and Longer Term Residents' Attitudes Toward Land Use, Development, and Environmental Issues in Rural Communities in Rocky Mountain West. Rural Sociology 65(3), p. 396-421.

Solana-Solana M., 2010. Rural gentrification in Catalonia, Spain: A case study of migration, social change and conflicts in the Empordanet area.,Geoforum, n 41, vol. 3, p. 508-517.

Subra P., 2006. Ce que le débat public nous dit du territoire et de son aménagement. Géocarrefour, n 4, vol. 81, p. 287-298.

Veschambre V., 2006. Penser l'espace comme dimension de la société, Pour une géographie sociale de plain-pied avec les sciences sociales, in Séchet R., Veschambre V. (dir.). Penser et faire la géographie sociale, Nouvelle édition [en ligne]. Rennes, PUR, p. 211-227. 